ТP Periodica Polytechnica Civil Engineering

\author{
62(2), pp. 404-412, 2018 \\ https://doi.org/10.3311/PPci.9650 \\ Creative Commons Attribution (i)
}

RESEARCH ARTICLE

\section{Numerical Simulation to Select Proper Strain Rates during CRS Consolidation Test}

\author{
Abderrahmane Henniche $^{1 *}$, Smain Belkacemi ${ }^{1}$
}

Received 22 June 2016; Revised 16 August 2017; Accepted 14 November 2017

\begin{abstract}
Constant rate of strain (CRS) and incremental loading (IL) consolidation tests are extensively used to measure consolidation properties of clayey soils. However, results of CRS test are usually strain rate dependent. In this study, a finite differences simulation of CRS test based on Terzaghi's theory of consolidation is performed. The numerical simulation permits to evaluate a strain rates range satisfying a preset relative pressure criterion. Results of simulation show that the required relative pressure criterion can be verified, during the steady state stage of CRS test, only for particular types of soils and particular range of strain rates. Subsequently, a simple method is proposed to select, for a soil sample defined by its initial height, initial void ratio and liquid limit, an appropriate strain rates range satisfying the ASTM 4186-06 criterion. Comparison of previsions and experimental results reported in literature shows good agreement.
\end{abstract}

\section{Keywords}

consolidation, CRS test, criteria, strain rate, liquid limit

\footnotetext{
1 Department of Civil Engineering,

Ecole Nationale Polytechnique,

10 Avenue of Hassen Badi, El Harrach, Algiers

* Corresponding author, email: henniche.lctp@yahoo.fr
}

\section{Introduction}

The constant rate of strain consolidation test (CRS test) was developed in 1959 by Hamilton and Crawford [1]. Its basic theory was developed by Smith and Wahls 1969 [2], and Wissa et al 1971 [3]. Constant rate of strain consolidation test reduces the required time for consolidation test using standard oedometer test (IL) from almost two weeks time to few hours. During CRS test the load is applied continuously; continuous record of stress and deformation are made, which improves the accuracy of consolidation properties determination in particular the preconsolidation pressure $P_{c}$.

Because of its many advantages, the CRS test is in many countries, a standard test for the determination of consolidation properties of clayey soils. It is adopted by the Swedish geotechnical institute, the Norwegian geotechnical institute, the French Laboratoire Central des Ponts et Chaussées (LCPC), and the American Society of Testing and Materials (ASTM) [4]. However, prior selection of a suitable strain rate for a given soil specimen, is still a problem to an extensive use of the CRS test in practice $[4,5]$, and many researches were conducted to examine strain rate effect on measured CRS consolidation parameters [6-9].

Comparison of CRS test results with those of conventional oedometer test has permitted to develop some criteria for the CRS test results acceptance. These criteria are essentially the relative pressure criterion $R_{u}[2,3,10]$, the liquid limit $L L$ criterion [11], and the standardized strain rate parameter $\beta$ based on the large strain theory $[4,12]$. The relative pressure criterion $R_{u}$ defined as the ratio of excess pore water pressure at the base of specimen $\Delta u_{H}$ to applied vertical stress $\Delta \sigma \mathrm{V}$ is generally the most used criterion. However, literature survey shows important differences in the acceptable range of relative pressure values. Table 01 summarizes some relative pressure values recommended by authors for some types of soils, with relative pressure range varying from 5 to $50 \%$. ASTM standard (ASTM 4186-06) recommends values of relative pressure to be between 3 and 15\% during steady state stage of CRS test [13]. Similar range of relative pressure is also recommended by the French Laboratory (LCPC) [14]. 
Table 1 Values of relative pressure recommended by authors [15]

\begin{tabular}{lcc}
\hline$R_{u}(\%)$ & Types of soils & References \\
\hline 50 & kaolinites, Ca-montmorillonites, & Smith and Wahls \\
Massena clay & (1969) \\
5 & $\begin{array}{c}\text { Boston blue clay } \\
\text { (sedimented artificially) }\end{array}$ & Wissa et al (1971) \\
$10-15$ & Bakebol Clay & Sällfors (1975) \\
$30-50$ & $\begin{array}{c}\text { Silts and clays from the coalfield of } \\
\left(u_{H, \min }=7 \mathrm{kPa}\right)\end{array}$ & Mississipi plains (Kentucky) \\
\hline
\end{tabular}

Based on empirical correlations, Gorman et al (1978) made general recommendations for the selection of the strain rate in terms of liquid limit $L L$, they recommended to conduct the CRS consolidation test at a strain of $5 \times 10^{-5} / \mathrm{min}$ for soils characterized by $L L>60 \%$, and for lower liquid limits the strain can be doubled [11].

Lee et al (1993) provided detailed estimation of parameters variation within the test specimen. Based on moving boundary and large strain theory, they defined a dimensionless strain $\beta=r . H_{0}^{2} / c_{v}$ to choose adequate strain rate $r$; where $H_{0}$ is the initial height of specimen, and $c_{v}$ is the coefficient of consolidation of soil. They recommended that the strain rate of CRS testing should have an upper bound of $\beta=0.1[4,12]$.

The proposed criteria lead to large differences in CRS strain rates estimates $[4,16]$. Authors $[17,18]$ have noted that this difference is probably due to the assumptions used in the CRS consolidation theory, in particular the applicability of Darcy's law.

CRS strain rate estimate still requires appropriate tools. This work constitutes a contribution to select appropriate strain rates during CRS test. A finite differences simulation of the CRS test, based on Terzaghi's theory, is performed to define an appropriate range of strain rates that satisfy a preliminary required relative pressure criterion, for specified soil characterized by constant value of $c_{v}$ (or LL), initial sample height $H_{0}$ and initial void ratio $e_{0}$.

\section{Theoretical aspect of CRS consolidation test}

CRS consolidation test results allow the evaluation of main soil consolidation and stress-strain characteristics such as the coefficient of consolidation $c_{v}$, hydraulic conductivity $k_{v}$, the preconsolidation stress $P_{c}$, and compression and recompression indexes $\left(c_{c}\right.$ and $\left.c_{r}\right)$.

The two most popular theories of CRS consolidation test are the linear theory of Smith and Wahls (1969), and the nonlinear theory of Wissa et al (1971). For small relative pressure values, the two theories are equivalent $[3,11]$.

Linear theory of Smith and Wahls (1969) assumes a constant coefficient of volume change $\left(m_{v}=\Delta \varepsilon_{v} / \Delta \sigma_{v}^{\prime}\right)$, a constant coefficient of permeability $k_{v}$, and a linear variation of void ratio $e$ within the specimen [2]. Based on these assumptions, they developed equations for average effective stress $\sigma_{v}^{\prime}$, hydraulic conductivity $k_{v}$ and coefficient of consolidation $c_{v}$.

$$
\begin{gathered}
\sigma_{v}^{\prime}=\sigma_{v 0}^{\prime}+\Delta \sigma_{v}-\alpha \cdot \Delta u_{H} \\
k_{v}=\frac{\gamma_{w} \cdot r \cdot H_{0}^{2}}{\left(1+e_{a v g}\right) \cdot \Delta u_{H}}\left(\frac{1}{2}-\frac{b}{12 r}\right) \\
c_{v}=\frac{H_{0}^{2}}{\Delta u_{H}}\left(\frac{\Delta \sigma_{v}}{\Delta t}\right)\left(\frac{1}{2}-\frac{b}{12 r}\right)
\end{gathered}
$$

$\sigma_{v 0}^{\prime}=$ initial effective stress

$\alpha=$ constant that takes generally values between 0.667 and 0.75 depending on the value of the constant $b / r$ (that takes generally values between 0 and 1 )

$b=$ constant that depends on variation of void ratio with depth and time

$\gamma_{w}=$ unit weight of water

$e_{\text {avg }}=$ average void ratio of specimen

$\Delta t=$ step time.

The theory of Wissa et al (1971) is based on the nonlinear consolidation theory of Davis and Raymond (1965) [19], it assumes a constant value of $c_{v}$ and a constant value of compression index $c_{c}$ (i.e., $d \varepsilon / d \log \sigma_{v}^{\prime}=$ constant). The solution consists of transient conditions that usually occur at the early stage of loading in which large excess pore water pressures are generated, and steady state conditions corresponding to a constant strain distribution. A dimensionless time factor $T$ was derived for CRS conditions, it indicates the degree of transience in the specimen strain distribution, and is evaluated from a function F3 that at any time equal to

$$
F_{3}=\frac{\log \left(\Delta \sigma_{v}+\sigma_{v 0}^{\prime}-\Delta u_{H}\right)-\log \sigma_{v 0}^{\prime}}{\log \left(\Delta \sigma_{v}+\sigma_{v 0}^{\prime}\right)-\log \sigma_{v 0}^{\prime}}
$$

A regression analysis of dimensionless time $T$ versus $F_{3}$ plot gives a simplified equation for $T$ [7], as:

$$
T=4.78\left(F_{3}\right)^{3}-3.21\left(F_{3}\right)^{2}+1.63 F_{3}+0.0356
$$

When $T<0.5$ the transient state is assumed, and when $T>$ 0.5 the steady state is assumed. The transition between these two phases is independent of the strain rate [3].

At the steady state conditions, the modified linear solution of Wissa et al (1971) theory is identical to that of Smith and Wahls (1969) with $b / r$ equal to zero, and the nonlinear solution of Wissa et al (1971) yields to these expressions:

$$
\begin{gathered}
\sigma_{v}^{\prime}=\left(\sigma_{v}^{3}-2 \cdot \sigma_{v}^{2} \cdot \Delta u_{H}+\sigma_{v} \cdot \Delta u_{H}^{2}\right)^{1 / 3} \\
k_{v}=-\frac{0.434 \cdot r \cdot H_{0}^{2} \cdot \gamma_{w} \cdot \Delta t}{2 \cdot \sigma_{v, a v g}^{\prime} \cdot \log \left(1-\frac{\Delta u_{H}}{\sigma_{v}}\right)} \\
c_{v}=-\frac{H_{0}^{2} \log \left(\frac{\sigma_{v 2}}{\sigma_{v 1}}\right)}{2 \cdot \Delta t \cdot \log \left(1-\frac{\Delta u_{H}}{\sigma_{v}}\right)} \\
\sigma_{v}=\sigma_{v 0}^{\prime}+\Delta \sigma_{v}
\end{gathered}
$$


$\sigma_{v, \text { avg }}^{\prime}$ in Eq.(7) is the average effective stress between two instants $t_{1}$ and $t_{2}$, with $\Delta t=t_{2}-t_{1}$ is the step time.

The two theories assume a parabolic distribution of pore water pressure along the specimen. If the specimen is deformed at a very low strain rate, very low or no pore pressure is generated, which affect the evaluation of the coefficient of consolidation cv. If the pore water pressure becomes excessive, evaluation of effective stress may be affected $[3,11]$. Therefore, the two theories proposed empirical methods that set the maximum strain rate by limiting the relative excess pore pressure $R_{u}$.

The ASTM (D4186-06) is generally the most used standard to conduct the CRS test. The ASTM standard was originally approved and published as D4186-82 in 1982. This test method applies to intact, remolded, or reconstituted soil samples. It is most often used for soils of relatively low hydraulic conductivity that allow generation of measurable excess pore pressures at the base of samples. In this test method the saturated specimen is constrained axially between two parallel, rigid platens, and without any lateral deformation. The minimum diameter and height of test specimen shall be about $50 \mathrm{~mm}$ and $20 \mathrm{~mm}$ respectively, and the maximum height-to-diameter ratio shall be 0.5 . A back pressure is applied to saturate both the specimen and the base pressure measurement system. A standard test includes one loading phase, one constant load phase, and one unloading phase. Because the stress-strain results of CRS consolidation tests are strain rate dependent, this test method limits the strain rates by specification of the excess pore pressure ratio $R_{u}$. This specification provides comparable results with standard consolidation test. In this test method, the rate of deformation is selected to produce an excess pore pressure ratio $R_{u}$ that will be between $3 \%$ and $15 \%$ in the normally consolidated range during the loading phase of the test. The equations in this test method are used only in steady state conditions $\left(F_{3}>0.4\right)$. Solutions for CRS consolidation in this test method are available for both linear and nonlinear soil models.

\section{Methodology of simulation}

Many studies [12, 20, 21] consider that the small strain theory can be only used to simulate the CRS consolidation test for small strain levels, and for large strain levels, significant differences in results between small and large strain theories are observed. These differences between small and large strain theories occur as a result of a reduction of specimen height during consolidation [21, 22, 23]. However, as the height of sample is known at any time during CRS test, the numerical solution of consolidation under constant loading (Terzaghi's theory) can be used iteratively to simulate the CRS consolidation test at small and large strains. Therefore, for successive very small time steps $\Delta t$, the additional loading between times $t$ and $t+\Delta t$ during CRS test, is considered temporary constant and equal to the difference:

$$
\Delta \sigma_{v}(t+\Delta t)-\Delta \sigma_{v}(t)
$$

Where $\Delta \sigma_{v}(t+\Delta t)$ and $\Delta \sigma_{v}(t)$ are the applied vertical load values during CRS test at times $t+\Delta t$ and $t$ respectively.

Terzaghi's equation of consolidation is expressed as [24]:

$$
c_{v} \frac{\partial^{2} u}{\partial z^{2}}=\frac{\partial u}{\partial t}
$$

At any time during CRS test, the height of sample $h(t)$ is divided into $n$ equal soil elements of thickness $\Delta z(t)$, so that:

$$
h(t)=H_{0}(1-r t)
$$

And,

$$
\Delta z(t)=h(t) / n
$$

Let $u_{r}, t_{r}$ and $z_{r}$ be any arbitrary reference excess pore water pressure, time, and depth, respectively. From these, the following dimensionless terms can be defined:

Dimensionless pore water pressure: $\bar{u}=u / u_{r}$

Dimensionless applied vertical load: $\Delta \bar{\sigma}_{v}=\Delta \sigma_{v} / u_{r}$

Dimensionless time: $\bar{t}=t / t_{r}$ and $\Delta \bar{t}=\Delta t / t_{r}$

Dimensionless depth: $\bar{z}=z / z_{r}$ and $\Delta \bar{z}=\Delta z / z_{r}$

The equation of consolidation becomes:

$$
\frac{u_{r}}{t_{r}} \frac{\partial \bar{u}}{\partial \bar{t}}=c_{v} \frac{u_{r}}{z_{r}^{2}} \frac{\partial^{2} \bar{u}}{\partial \bar{z}^{2}}
$$

We can consider that: $t r=z_{r}^{2} / c_{v}$, then Eq.(13) will be of the form :

$$
\frac{\partial \bar{u}}{\partial \bar{t}}=\frac{\partial^{2} \bar{u}}{\partial \bar{z}^{-2}}
$$

The left-hand and right-hand sides of Eq.(14) can be written as:

$$
\frac{\partial \bar{u}}{\partial \bar{t}}=\frac{1}{\Delta \bar{t}}\left(\bar{u}_{\bar{z}, \bar{t}+\Delta \bar{t}}-\bar{u}_{\bar{z}, \bar{t}}\right)
$$

Where $\bar{u}_{\bar{z}, \bar{t}+\Delta \bar{t}}$ and $\bar{u}_{\bar{z}, \bar{t}}$ are the dimensionless pore water pressures at the dimensionless depth $\bar{z}$, and at dimensionless times $\bar{t}+\Delta \bar{t}$ and $\bar{t}$ respectively (Fig.1).

$$
\frac{\partial^{2} \bar{u}}{\partial \bar{z}^{-2}}=\frac{1}{(\Delta \bar{z})^{2}}\left(\bar{u}_{\bar{z}-\Delta \bar{z}, i}+\bar{u}_{\bar{z}+\Delta \bar{z}, \bar{i}}-2 \bar{u}_{\bar{z}, \bar{i}}\right)
$$

Where $\bar{u}_{\bar{z}-\Delta \bar{z}, \bar{t}}$ and $\bar{u}_{\bar{z}+\Delta \bar{z}, \bar{t}}$ are the dimensionless pore water pressures at the dimensionless time, $\bar{t}$ and at dimensionless depth $\bar{z}-\Delta \bar{z}$ and $\bar{z}+\Delta \bar{z}$ respectively (Fig.1).

Substituting Eqs.(15) and (16) into Eq.(14), leads to:

$$
\bar{u}_{\bar{z}, \bar{i}+\Delta \bar{t}}=\frac{\Delta \bar{t}}{(\Delta \bar{z})^{2}}\left(\bar{u}_{\bar{z}-\Delta \bar{z}, \bar{t}}+\bar{u}_{\bar{z}+\Delta \bar{z}, \bar{i}}-2 \bar{u}_{\bar{z}, \bar{i}}\right)+\bar{u}_{\bar{z}, \bar{i}}
$$

For Eq. (17) to converge, $\Delta \bar{t}$ and $\Delta \bar{z}$ must be chosen such that:

$$
\Delta \bar{t} /(\Delta \bar{z})^{2}<0.5
$$

To consider the height of specimen $h(t)$ at any time during CRS test, the arbitrary reference depth is taken equal to $z_{r}=h(t)$, Then Eq.(18) will be of the form : 


$$
\Delta \bar{t} /(\Delta \bar{z})^{2}=\Delta t \cdot c_{v} \cdot n^{2} /(h(t))^{2}
$$

The value of $\Delta t$ is evaluated in terms of a preset final strain of specimen $\varepsilon_{\max }$. If pore water pressure converges for $\Delta t$ and $\Delta z$ estimated from $\varepsilon_{\max }$, it converges automatically during all the test duration.

For an undrained base of specimen, we take: $\bar{u}_{\bar{z}-\Delta \bar{z}, \bar{t}}=\bar{u}_{\bar{z}+\Delta \bar{z}, \bar{t}}$, and Eq.(17) becomes:

$$
\bar{u}_{\bar{z}, \bar{i}+\Delta i}=\frac{\Delta \bar{t}}{(\Delta \bar{z})^{2}}\left(2 \bar{u}_{\bar{z}-\Delta \bar{z}, \bar{i}}-2 \bar{u}_{\bar{z}, \bar{i}}\right)+\bar{u}_{\bar{z}, \bar{i}}
$$

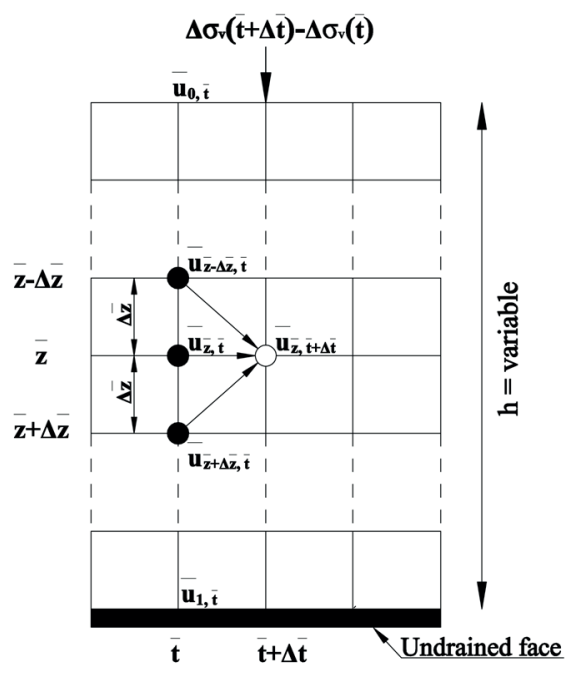

Fig. 1 Numerical solution of consolidation algorithm.

Due to continuous loading during CRS test, an additional load at each time step must be considered in Eq.(17). The variation of applied vertical load versus time will be estimated from variation of effective stress versus time for normally consolidated soils by considering the pore water pressure buildup along the specimen. The void ratio-effective stress relation for normally consolidated soils is assumed linear in semi logarithmic space:

$$
e=e_{0}-c_{c} \log \left(\sigma_{v}^{\prime} / \sigma_{v 0}^{\prime}\right)
$$

Where:

$c_{c}=$ the compression index assumed constant

$e_{0}=$ the initial void ratio of soil subjected to an initial effective stress $\sigma_{v 0}^{\prime}$.

For CRS test, variation of void ratio versus time is given by [17]:

$$
\begin{gathered}
e=e_{0}-C \times t \\
C=\left(1+e_{0}\right) \times r
\end{gathered}
$$

The variation of effective stress versus time, in semi logarithmic space, for CRS test is expressed as:

$$
\sigma_{v}^{\prime}=\sigma_{v 0} \times 10^{\left(C \times t / c_{c}\right)}
$$

And the effective stress change at time $t$ is:

$$
\Delta \sigma_{v}^{\prime}=\sigma_{v 0}^{\prime} \times\left(10^{\left(C \times t / c_{c}\right)}-1\right)
$$

The effective stress change is essentially due to the applied vertical stress change. At each iteration of CRS test, the total applied stress $\Delta \sigma_{v}(t+\Delta t)$ at time $t+\Delta t$, is the sum of the effective stress change at time $t+\Delta t$, and the average pore water pressure along the specimen noted $\operatorname{uavg}(t+\Delta t)$ at time $t+\Delta t$ :

$$
\Delta \sigma_{v}(\mathrm{t}+\Delta \mathrm{t})=\Delta \sigma_{v}^{\prime}(\mathrm{t}+\Delta \mathrm{t})+u_{\text {avg }}(\mathrm{t}+\Delta \mathrm{t})
$$

At the beginning of each iteration at time $t+\Delta t$, because the value of $u_{\text {avg }}(t+\Delta t)$ is unknown, we use the value of $u_{\text {avg }}(t)$ evaluated at time t to calculate $\Delta \sigma_{v}(t+\Delta t)$ at time $t+\Delta t$. The obtained value of $\Delta \sigma_{v}(t+\Delta t)$ is used to evaluate uavg $(t+\Delta t)$ by Eq.(29). The new value of $u_{\text {avg }}(t+\Delta t)$ will be used to calculate again $\Delta \sigma_{v}(t+\Delta t)$ at time $t+\Delta t$. This procedure is repeated iteratively at time $\mathrm{t}+\Delta \mathrm{t}$ until the difference between two subsequent values of $\Delta \sigma_{v}(t+\Delta t)$ is less than a tolerance.

The estimated total applied stress at time $(t+\Delta t)$ is then given by:

$$
\Delta \sigma_{v}(t+\Delta t)=\sigma_{v 0}^{\prime}\left(10^{\left(C \times(t+\Delta t) / c_{c}\right)}-1\right)+u_{\text {avg }}(t+\Delta t)
$$

The average pore water pressure of specimen at time $t+\Delta t$, $u_{\text {avg }}(t+\Delta t)$ is equal to:

$$
u_{\text {avg }}(t+\Delta t)=\frac{1}{H} \int_{0}^{H} u_{z, t+\Delta t} \cdot d z
$$

Eq.(28) is numerically evaluated by the trapezoidal integration method.

The effect of total applied stress between $t$ and $t+\Delta t$ that equal to $\Delta \sigma_{v}(t+\Delta t)-\Delta \sigma_{v}(t)$, is added to Eq.(17) to estimate the distribution of pore water pressure $\bar{u}_{\bar{z}, \bar{t}+\Delta \bar{t}}$ :

$$
\begin{gathered}
\bar{u}_{\bar{z}, \bar{t}+\Delta \bar{t}}=\frac{\Delta \bar{t}}{(\Delta \bar{z})^{2}}\left(\bar{u}_{\bar{z}-\Delta \bar{z}, \bar{t}}+\bar{u}_{\bar{z}+\Delta \bar{z}, \bar{i}}-2 \bar{u}_{\bar{z}, \bar{t}}\right)+\bar{u}_{\bar{z}, \bar{i}}+I \\
I=\frac{\Delta \sigma_{v}(\bar{t}+\Delta \bar{t})-\Delta \sigma_{v}(\bar{t})}{u_{r}} \\
u_{\bar{z}, \bar{t}+\Delta \bar{t}}=\bar{u}_{\bar{z}, \bar{i}+\Delta \bar{t}} \times u_{r}
\end{gathered}
$$

For CRS test, at $t=0, \Delta \sigma_{v 0}^{\prime}=0, \Delta u_{H}=0$ and $\Delta \sigma_{v}=0$.

This numerical simulation includes the following inputs:

Initial specimen height $\boldsymbol{H}_{\mathbf{0}}$ : Soil specimen heights in CRS consolidation tests are generally ranging from 20 to $25 \mathrm{~mm}$. In this simulation, the specimen height of $25 \mathrm{~mm}$ is used; and for comparisons with experimental works, heights of tested samples are considered in calculations.

Coefficient of consolidation $c_{v}$ and compression index $c_{c}$ : are evaluated using the soil liquid limit $L L$. The value of coefficient of consolidation $c_{v}$, is estimated for normally consolidated undisturbed soils (Fig.02); and the value of compression index is evaluated according to the Terzaghi and Peck's correlation [25]:

$$
c_{c}=0.009(L L-10)
$$

Initial void ratio $e_{0}$ : to evaluate the effect of initial void ratio on the results, values ranging from 0.2 to 3.0 are considered. 


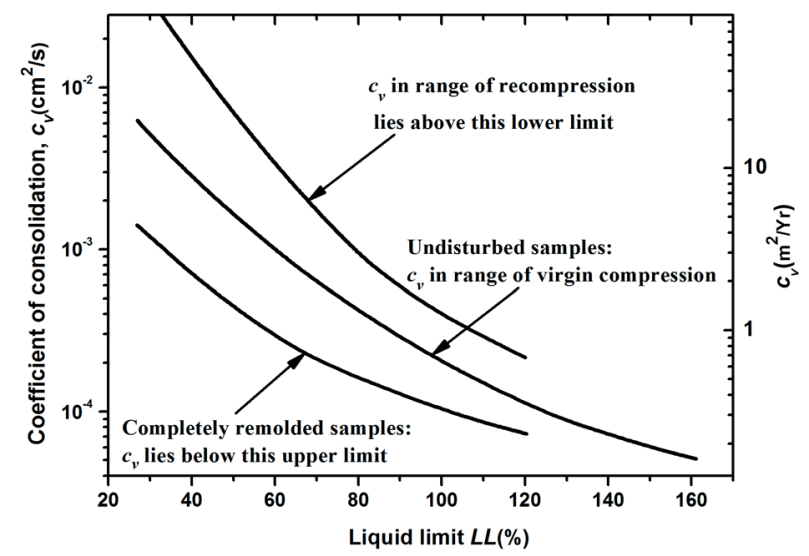

Fig. 2 Approximate correlations between coefficient of consolidation $c_{v}$ and liquid limit $L L[26]$.

Initial effective stress $\sigma_{v 0}^{\prime}$ : Only the lowest and largest possible values of initial effective stress are considered in this simulation. The lowest value of $15 \mathrm{kPa}$ permits to obtain the lower limit of strain rates range, and the largest value of $100 \mathrm{kPa}$ permits to obtain the upper limit of strain rates range. The strain rates range obtained (common range) will satisfy the required criterion for all other values of initial effective stress bounded by 15 and $100 \mathrm{kPa}$.

Number of specimen elements $\boldsymbol{n}$ : different conducted simulations indicate that results converge for $n$ varying from 20 to 100 .

Total time of test: calculations are stopped when the applied stress is greater than a preset value $\Delta \sigma_{v, \max }$, or when a specified value of final average strain $\varepsilon_{\max }$ is reached. In this simulation, calculations are stopped if $\Delta \sigma_{v} \geq 2.0 \mathrm{MPa}$ or if $\varepsilon_{\max } \geq 40 \%$; and during comparisons with experimental works, the maximum strain or load reached during testing are considered.

The different parts of structure model can be illustrated by the flow chart in Fig.03.

\section{Results of simulation}

For a soil characterized by constant values of coefficient of consolidation $c_{v}$ and compression index $c_{c}$, the strain rates are varied from the smallest to the largest $\left(10^{-6} / \mathrm{min}\right.$ to $\left.10^{-2} / \mathrm{min}\right)$. For each value of strain rate, the variation of relative pressure $R_{u}$ versus time is evaluated (Fig.04) and the following results are observed:

- The initial load is essentially taken by pore water, and very high levels of relative pressure are produced $\left(R_{u}=1\right)$. After very short time, values of relative pressure decrease sharply for any strain rate. For a short period of time, the relative pore pressure is independent of strain rate. This stage corresponds to the transient state of CRS test. As mentioned by Wissa et al (1971), the transition from the transient state to the steady state is independent of the strain rate.

- At the start of the steady state, and also for a short period of time, the relative pressure still decrease sharply at a rate dependent on strain rate. At the remaining time of steady state, low rate of relative pressure decrease is observed. During all the steady state, the strain rate effect is important, and values of relative pressure increase with strain rates.

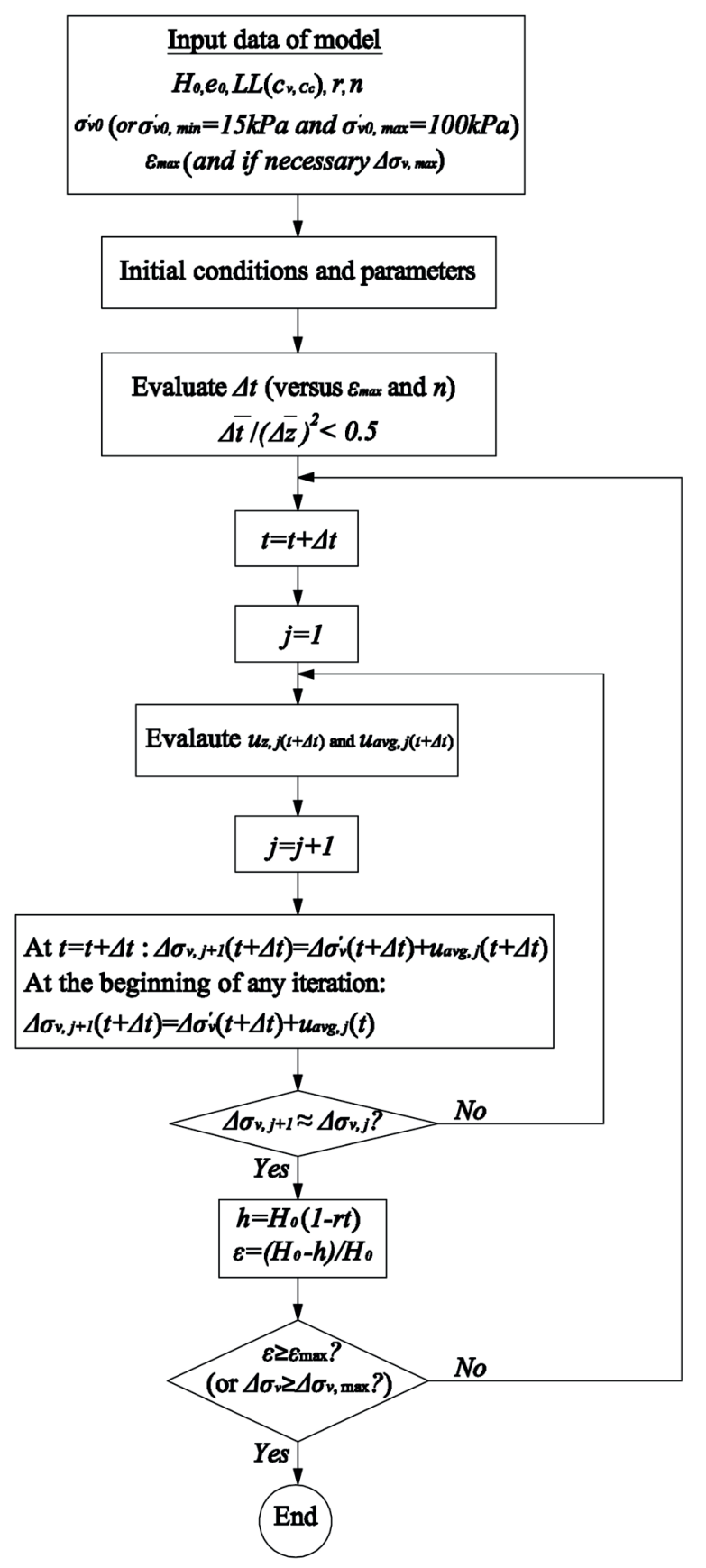

Fig. 3 Flow chart of the numrical simulation.

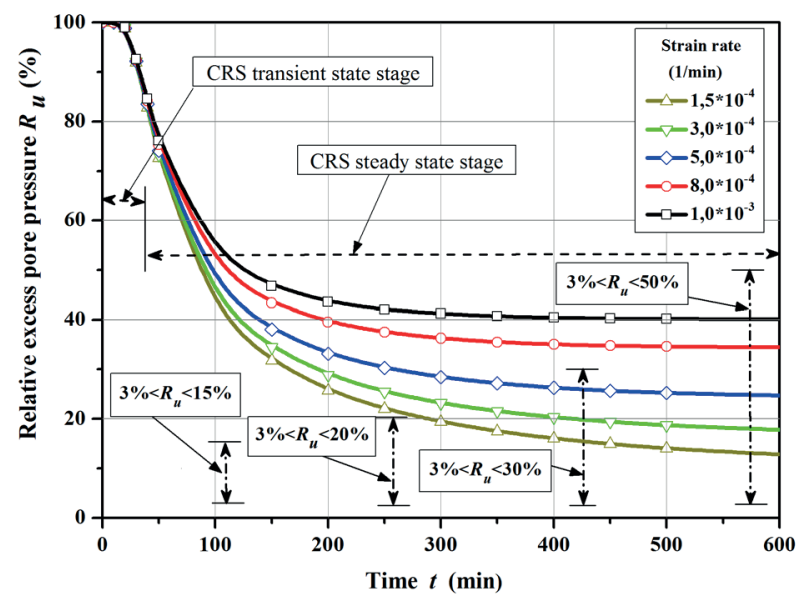

Fig. 4 Variations of relative pore pressure versus time, $\left(c_{v}=0.06 \mathrm{~cm}^{2} / \mathrm{min}, e_{0}=1.2, H_{0}=25 \mathrm{~mm}\right)$. 
The variations of relative pressure $R_{u}$ versus time, for a soil characterized by coefficient of consolidation $c_{v}=0.06 \mathrm{~cm}^{2} /$ min, void ratio $e_{0}=1.2$, initial sample height of $25 \mathrm{~mm}$, and for a strain rates ranging from $1.5 \times 10^{-4} / \mathrm{min}$ to $1 \times 10^{-3} / \mathrm{min}$, are represented in Fig. 04.

Measured values of relative pore pressure in the initial phase of test are often lower than one $\left(R_{u}<1\right)$. This is may be due to either lack of saturation, the compressibility of the pore water or to the rigidity of the soil skeleton. With the decrease in soil porosity and an increase in the quasi-saturation of the pores, the distribution of pore water pressure in the subsequent part of the test is consistent with the theoretical results [15].

Simulation results show that in the transient stage and at the start of the steady state, and for any strain rate, the required relative pressure criteria cannot be satisfied. However, for a substantial duration of the steady state stage, the required relative pressure criteria, for any strain rate, can be satisfied. In this simulation, strain rates, for which average value of relative pressure in the steady state stage satisfying the relative pressure criterion, are evaluated.

For a given initial void ratio $e_{0}$, the extent of the appropriate strain rates range depends on the value of the coefficient of consolidation $c_{v}$ (or $L L$ ), and the specified relative pressure criterion. The ranges of appropriate strain rates versus coefficient of consolidation $c_{v}$ (or $L L$ ) for various relative pressure requirements, for initial void ratio of 1.2 , and initial sample height of $25 \mathrm{~mm}$ are shown in Fig.05.

To obtain CRS consolidation parameters similar to those obtained in IL tests, ASTM standard (ASTM D4186-06) recommends choosing appropriate strain rates such as relative pressure values during steady state are between 3 and $15 \%$. Appropriate strain rates versus liquid limit, for an initial sample height of $25 \mathrm{~mm}$ and for various void ratios - from 0.2 to 3.0-, that satisfy ASTM relative pressure criterion are presented in Fig.06.

It is observed that for a soil sample with an initial void ratio $e_{0}$ and with a liquid limit $L L \leq 90 \%$, appropriate strain rates satisfying the ASTM standards criterion can be obtained. To satisfy ASTM criterion, soils with lower initial void ratios or liquid limits require higher strain rates than soils with higher void ratios or liquid limits.

\section{Simulation verification}

To check the performance of this numerical simulation, predicted results are compared with some experimental works reported in literature (Gorman et al 1978, and T.M.H. Lok, X. Shi 2008). Proper strain rates predicted by this numerical simulation are also compared with Gorman et al and ASTM D4186-06 recommendations.
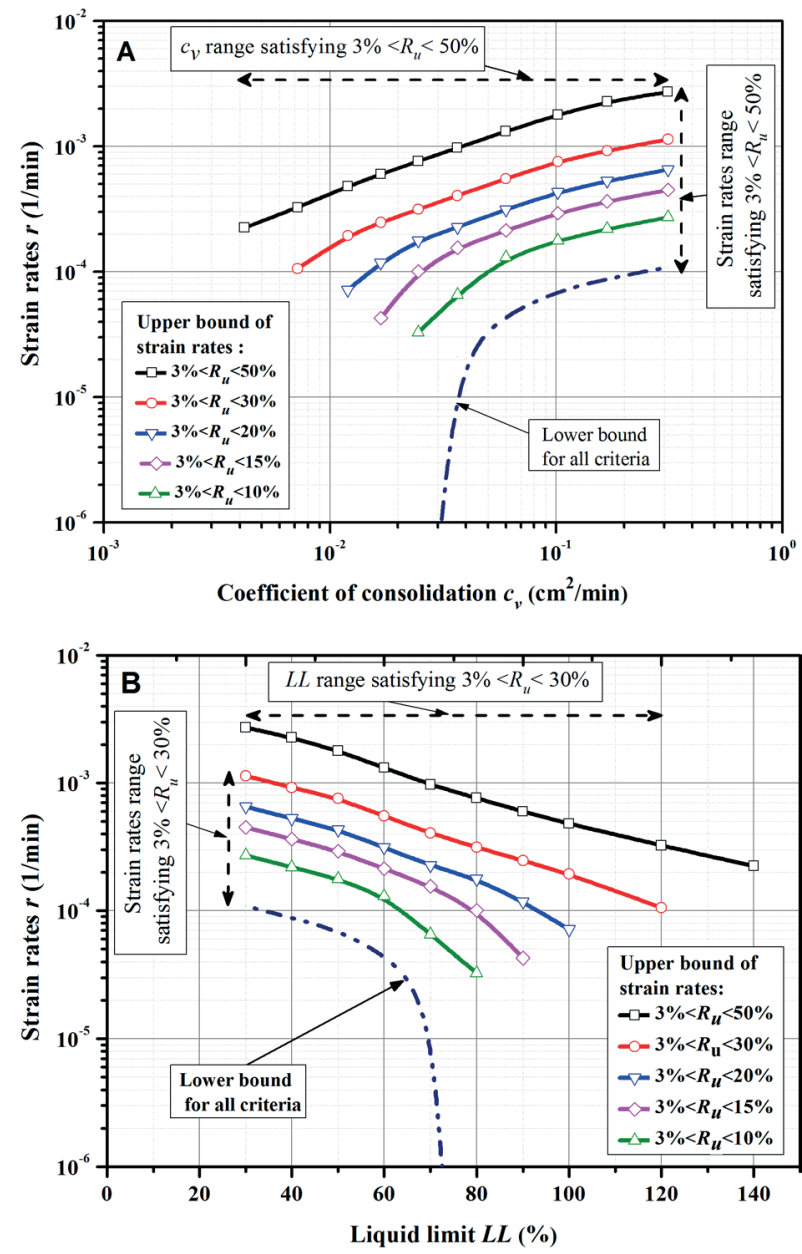

Fig. 5 Extents of appropriate strain rates satisfying various required relative pressures criteria $\left(c_{v}=0.06 \mathrm{~cm}^{2} / \mathrm{min}, e_{0}=1.2, H_{0}=25 \mathrm{~mm}\right)$.
A) versus $c$.
B) versus $L L$.

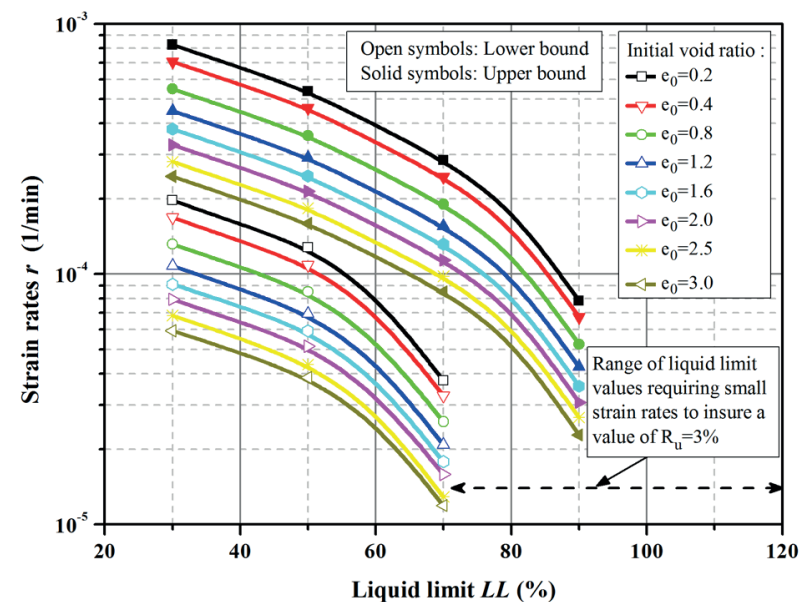

Fig. 6 Proper strain rates satisfying ASTM criterion $\left(3 \%<R_{u}<1 \%\right)$ versus liquid limit and initial void ratio $\left(H_{0}=25 \mathrm{~mm}\right)$.

\section{Case 01: Experimental results of Gorman et al [11].}

In 1977, Gorman et al conducted various types of consolidation tests (Incremental Loading IL, Controlled Gradient CG and Constant Rate of Strain CRS tests) on intact soil samples from three sites of Kentucky. The undisturbed samples were taken at each $1.5 \mathrm{~m}$ intervals from sites one and two, and continuously at site three. Each test specimen was trimmed to $64 \mathrm{~mm}$ diameter by $25 \mathrm{~mm}$ height. A back pressure of $69 \mathrm{kPa}$ 
was applied in all CG and CRS tests at top and bottom of the specimen, and monitored for at least 12 hours before testing.

The incremental tests were performed in accordance with ASTM D 2435-70 standards. Drainage was allowed at top and bottom of specimen. Load increments of $(0.025,0.05,0.1,0.2$, $0.4,0.8,1.6,3.2 \mathrm{MPa})$ were applied. Each load increment was maintained constant on the specimen for 24 hours before the application of the next increment.

For CRS tests, the specimen was deformed by a constant predetermined rate. The loading was stopped at a preset load of $3 \mathrm{MPa}$ and the pore water pressure was allowed to dissipate at the final, constant load. This loading level produced different final strains of specimens ranging from $12 \%$ to $30 \%$. The specimen was finally unloaded at the same rate at which it was loaded. Readings of load, pore pressure and height of specimen, were taken at various time intervals of test.

A good agreement between IL, CRS and CG compressibility curves and $c_{v}$ variations is obtained for site 02 , and some scatter was shown for sites 01 and 03 , but generally no significant differences were observed in IL, CRS and CG test data above the preconsolidation pressure. During CRS tests the imposed strain rates induced maximum values of relative pressures that vary in most tests between 3 and 15\%.

Different simulations were conducted to estimate, for all CRS tests, a strain rates range conforming to the ASTM standards criterion $\left(3 \%<R_{u}<15 \%\right)$. For the sake of comparison, values of initial void ratio of different soil samples were not indicated by authors, in this study it was deduced that the values of initial void ratio are in general within the range of $(0.4-1.2)$. The bounds of predicted range correspond to $e_{0}=0.4$ and $e_{0}=1.2$. Calculations were stopped when the applied load or vertical strain reaches respectively $3 \mathrm{MPa}$ or $30 \%$. Strain rates used during CRS tests are generally found within the predicted appropriate range of strain rates that comply with ASTM standard and for initial void ratios that are between 0.4 and 1.2 (Fig.07). Gorman et al concluded that in CRS test, a considerable range of strain rates could be used for the same type of soil without significant difference in results. Similar results were obtained on two identical soil samples tested at different strain rates $\left(3.8 \times 10^{-5} / \mathrm{min}\right.$ and $\left.1.6 \times 10^{-4} / \mathrm{min}\right)$. These strain rates are also within the predicted range (Fig.07).

Case 02: Experimental results of T.M.H. Lok and X. Shi

T.M.H. Lok and X. Shi [27] performed IL and CRS consolidation tests on two types of Macau marine clays, the first is reconstituted characterized by $L L=65$ and $e_{0}=1.65$, and deformed under strain rate of $2 \% / \mathrm{h}$, the second is undisturbed characterized by $L L=60$ and $e_{0}=1.37$, and consolidated using a strain rate of $1 \% / \mathrm{h}$.

A consolidometer was used to prepare the reconstituted samples. The material necessary to prepare the reconstituted samples was taken from an excavation site in Macau, and was then mixed into thick slurry and passed through $600 \mu \mathrm{m}$

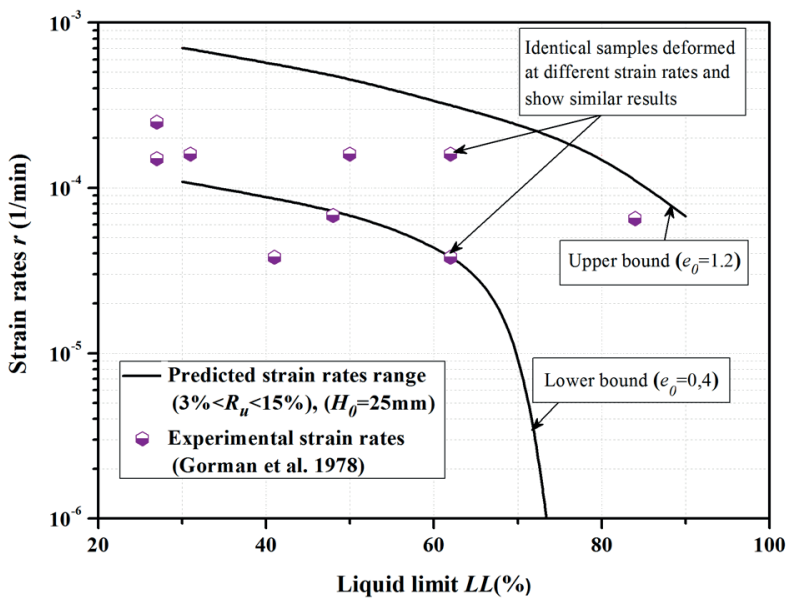

Fig. 7 Comparison between imposed strain rates (Gorman et al. 1978) and simulation results $\left(3 \%<R_{u}<15 \%\right)$.

standard sieve to remove all large soil particles and shells. The slurry was poured into the consolidometer for consolidation, and a pressure of lbar was applied to the slurry from the bottom cap. The consolidation was stopped when the primary consolidation had finished. After finishing the consolidation, the sample was carefully pushed out by hydraulic extruder and was cut into different sizes depending on the tests to be performed, but the peripheral part of the specimen was discarded because of disturbance.

Two different sizes of undisturbed samples were obtained with $76 \mathrm{~mm}$ stainless steel Shelby piston tube sampler and U100 steel tube sampler with the length of $1 \mathrm{~m}$ and $0.5 \mathrm{~m}$, respectively. The undisturbed clay samples were taken at the depth of $3 \mathrm{~m}$ to $6 \mathrm{~m}$ from the first site (Taipa), and at the depth of $6 \mathrm{~m}$ to $13 \mathrm{~m}$ from the second site (Cotai). After the sample was taken out from the bore-hole, both ends of the sample tube were sealed with wax in the field.

In incremental tests, the cell of $75 \mathrm{~mm}$ in diameter was used to explore the consolidation behavior of reconstituted samples, while the cell of $50 \mathrm{~mm}$ in diameter was used to explore the behavior of undisturbed sample, and both cells have the height of $20 \mathrm{~mm}$. Each load increment is maintained constant for 24 hours, and the load was doubled for the next increment. During the consolidation process, the vertical displacements were recorded at time intervals of $0,0.1,0.25,0.5,1,2,4,8,15$, $30,60,120,240,480,720,1440$ minutes after the application of each load increment.

In CRS tests, during saturation phase a back pressure of $200 \mathrm{kPa}$ was applied at both sides of specimens having $20 \mathrm{~mm}$ height. Three CRS tests were performed; two with a strain rate of $2 \% / \mathrm{h}$ and one with a strain rate of $1 \% / \mathrm{h}$. Tests were stopped around $1500 \mathrm{kPa}$ for reconstituted samples and $1100 \mathrm{kPa}$ for undisturbed sample. Final strains of about $48 \%$ and $27 \%$ were reached respectively for reconstituted and undisturbed samples.

Estimates of strain rate ranges that comply with ASTM CRS test criterion versus liquid limits for sample height of $20 \mathrm{~mm}$ and void ratios of 1.37 and 1.65 are shown in Fig.08. 
Calculations were stopped for reconstituted samples when the applied load reaches $1500 \mathrm{kPa}$ or the vertical strain reaches $48 \%$ and for undisturbed sample when the applied load reaches $1100 \mathrm{kPa}$ or the vertical strain reaches $27 \%$.

Strain rate used for the reconstituted samples lies above the predicted strain range that complies with ASTM criterion. While the strain rate used for the undisturbed sample lies in the predicted strain range that complies with ASTM criterion (Fig.08). During CRS tests, the relative pressure reached $55 \%$ in reconstituted samples and it did not exceed $15 \%$ in the undisturbed sample. It is also worth noting that differences, in consolidation properties - coefficient of consolidation and preconsolidation pressure - obtained from CRS and IL tests, were larger for reconstituted than for undisturbed samples of Macau marine clays.

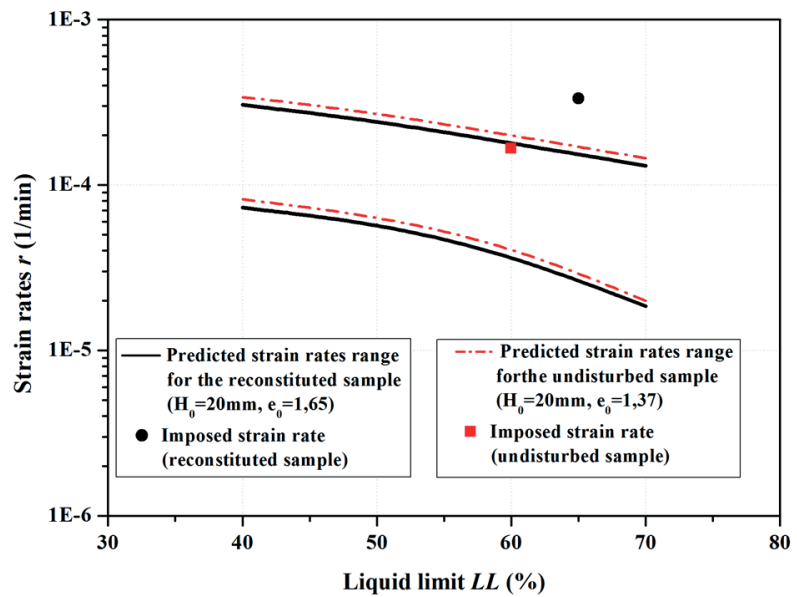

Fig. 8 Comparison between strain rates imposed on the Macau marine clays and simulation results $(3 \%<\mathrm{Ru}<15 \%)$.

Case 03: Comparison Gorman et al and ASTM D4186-06 recommendations

Gorman et al recommended to apply a strain rate of $50 \times 10^{-6} /$ min for soils characterized by $L L>60 \%$, and to double it for soils with $L L<60 \%$. Also, ASTM standards (ASTM D4186-06) recommends starting the CRS tests with a strain rate of $10 \% / \mathrm{h}$ $\left(1.66 \times 10^{-3} / \mathrm{min}\right)$ for $\mathrm{MH}$ soils type, a strain rate of $1 \% / \mathrm{h}(1.66 \times$ $\left.10^{-4} / \mathrm{min}\right)$ for the CL soils type, and a strain rate of $0.1 \% / \mathrm{h}(1.66$ $\times 10^{-5} / \mathrm{min}$ ) for the $\mathrm{CH}$ soils type. The comparison with the present numerical simulation results shows that (Fig.09):

For $\leq 50 \%$; both ASTM and Gorman et al recommendations fall in the predicted appropriate strain rates range for values of relative pressure such as $\left(3 \%<R_{u}<15 \%\right)$.

For $L L>50 \%$ and for the $\mathrm{CH}$ materials; both ASTM and Gorman et al recommendations fall in the predicted appropriate strain rates range for values of relative pressure such as $\left(3 \%<R_{u}<15 \%\right)$.

\section{Conclusions}

Numerical solution of consolidation for constant loading (Therzagi's theory) is used in this work to simulate the case of continuous loading of CRS consolidation test. The aim of

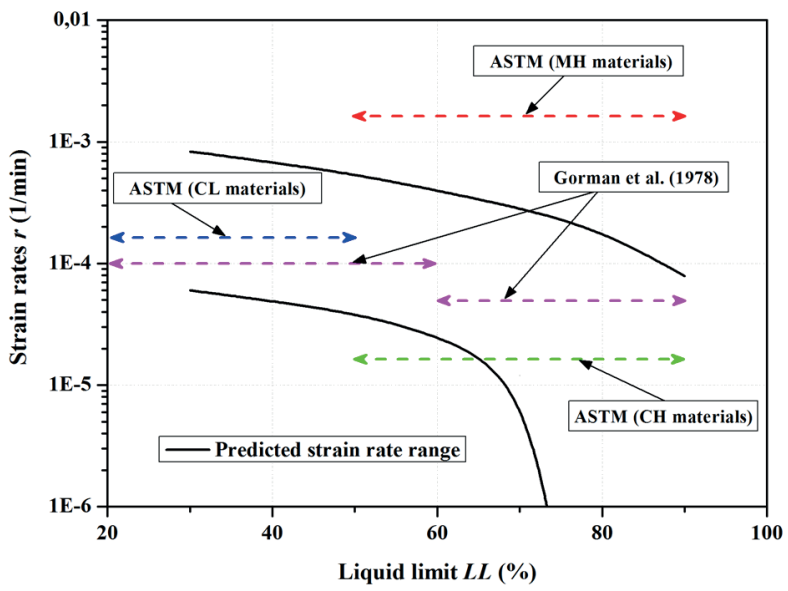

Fig. 9 Comparison between ASTM recommendations, recommendations of Gorman et al and simulation predictions $(3 \%<\mathrm{Ru}<15 \%)$.

this simulation is to evaluate appropriate ranges of strain rates satisfying various preset relative pressure criteria, and in particular ASTM standard criterion.

During simulation, the load-time relation during CRS test is assumed from the variation of effective stress versus time for normally consolidated soils and the average buildup of pore water pressure. Variation of effective stress versus time for normally consolidated soils is affected by strain rate, compression index and initial porosity of soils.

Correlations between limit liquid $L L$, coefficient of consolidation $c_{v}$ and compression index $c_{c}$ of normally consolidated soils are used during simulation. The initial void ratio is varied between 0.2 and 3.0 .

The results of this numerical simulation show the following:

The required relative pressure criteria cannot be verified for any strain rate throughout the duration of CRS test, but they can be verified for particular strain rates for a substantial duration of test.

For a required relative pressure criterion, there are ranges of liquid limits and appropriate strain rates that satisfy this criterion.

For a given soil specimen defined by its height, strain rates that satisfy the relative pressure criterion recommended by ASTM standard $\left(3 \%<R_{u}<15 \%\right)$ are strongly dependent on the value of initial void ratio of the sample and on the soil compressibility.

Comparison between simulation results and experimental results reported in literature indicates that simulation previsions are generally satisfactory, and the relative pressure criterion recommended by ASTM standard leads to CRS consolidation parameters comparable to those of IL test.

\section{References}

[1] Hamilton, J. J., Crawford, C. B. "Improved determination of preconsolidation pressure of a sensitive clay". Papers on Soils, ASTM STP 254, Committee D-18, Eds, ASTM International, West Conshohocken, PA, pp. 254-270. 1959. https://doi.org/10.1520/STP44323S

[2] Smith, R. E., Wahls, H. E. "Consolidation under constant rate of strain". Journal of the Soil Mechanics and Foundations Division, 95(2), pp. 519 539. 1969. 
[3] Wissa, A. E. Z., Christian, J. T., Davis, E. H., Heiberg, S. "Consolidation at constant rate of strain". Journal of the Soil Mechanics and Foundations Division, 97(10), pp. 1393-1413. 1971.

[4] Lee, K., Choa, V., Lee, S. H., Quek, S. H. "Constant rate of strain consolidation of Singapore Marine Clay". Geotechnique, 43(3), pp. 471-488. 1993. https://doi.org/10.1680/geot.1993.43.3.471

[5] Crawford, C. B. "On the Importance of Rate of Strain in the Consolidation Test". Geotechnical Testing Journal, 11(1), pp. 60-62. 1988. https:// doi.org/10.1520/GTJ10646J

[6] Leroueil, S., Kabbaj, M., Tavenas, F., Bouchard, R. "Stress-StrainStrain Rate Relation for the Compressibility of Sensitive Natural Clays". Geotechnique, 35(2), pp. 159-180. 1985. https://doi.org/10.1680/ geot.1985.35.2.159

[7] Sheahan, T. C., Watters, P. J. "Experimental Verification of CRS Consolidation Theory". Journal of Geotechnical and Geoenvironmental Engineering, 123(5), pp. 430-437. 1997. https://doi.org/10.1061/(ASCE)1090-0241 (1997)123:5(430)

[8] Adams, A. L. "Laboratory Evaluation of the Constant Rate of Strain and Constant Head Techniques for Measurement of the Hydraulic Conductivity of Fine Grained Soils". M.S. thesis, Massachusetts Institute of Technology, Cambridge, MA. 2011. http://www-udc.ig.utexas.edu/geofluids/ Theses/Adams_thesis.pdf

[9] Sample, K. M., Shackelford, C. D. "Apparatus for constant rate of strain consolidation of slurry mixed soils". Geotechnical Testing Journal, 35(3), pp. 409-419. 2012. https://doi.org/10.1520/GTJ103787

[10] Sallfors, G. "Preconsolidation pressure of soft highly plastic clays". Chalmers University of Technology, Goteborg. 1975.

[11] Gorman, C. T., Hopkins, T. C., Deen, R. C., Drnevich, V. P. "Constant Rate of Strain and Controlled Gradient Consolidation Testing". Geotechnical Testing Journal, 1(1), pp. 3-15. 1978. https://doi.org/10.1520/ GTJ10363J

[12] Lee, K. "Consolidation With Constant Rate of Deformation". Geotechnique, 31(2), pp. 215-229. 1981. https://doi.org/10.1680/ geot.1981.31.2.215

[13] ASTM. "Standard test method for one-dimensional consolidation properties of saturated cohesive soils using controlled-strain loading". D418606, West Conshohocken, PA. 2006. http://www.astm.org/Standards/ D4186

[14] Mieussens, C., Magnan, J. P., Soyez, P. "Essais de compressibilité à l'oedomètre, Procédures recommandées par les laboratoires des ponts et chaussées", Translation to English "Oedometer compressibility tests, Procedures recommanded by les laboratoires des ponts et chaussées". Bulletin de liaison des laboratoires des ponts et chaussées, $N^{\circ} 139.1985$. (In French)

[15] Dobak, P., Kowalczyk, S. "Evaluation of consolidation parameters in CL tests; theoretical and practical aspects". Geological Quarterly, 52 (4), pp. 397-410. 2008. https://gq.pgi.gov.p1/article/view/7501/6151

[16] Almeida, M. S. S., Martins, I. S., Carvalho, S. R .L. "Constant rate of strain consolidation of Singapore marine clay-discussion to paper". Geotechnique, 45(2), pp. 333-336. 1995. https://doi.org/10.1680/ geot.1995.45.2.333

[17] Ahmadi, H., Hoorfar, A. H., Rahimi. H., Soroush, A. "Hydraulic characteristics and pore water flow rule in constant rate of strain consolidation". American Journal of Applied Sciences, 6(7), pp. 1429-1435. 2009. https://doi.org/10.3844/ajassp.2009.1429.1435

[18] Ahmadi, H., Rahimi, H., Soroush, A., Claes, A. "Experimental research on variation of pore water pressure in constant rate of strain consolidation test". Acta Geotechnica Slovenica, 11(2), pp. 47-57. 2014.

[19] Davis, E. H., Raymond, G. P. "A non-linear theory of consolidation". Geotechnique, 15(2), pp. 161-173. 1965. https://doi.org/10.1680/ geot.1965.15.2.161
[20] Rui, J., Jinchun, C., Takenori, H. "Interpretation of coefficient of consolidation from CRS test results". Geomechanics and Engineering, 5(1), pp. 57-70. 2013. https://doi.org/10.12989/gae.2013.5.1.057

[21] Fox, P. J., Pu, H. "Enhanced CS2 Model for Large Strain Consolidation". International Journal of Geomechanics, 12(5), pp. 574-583. 2012. https://doi.org/10.1061/(ASCE)GM.1943-5622.0000171

[22] Pu, H., Fox, P. J., Liu, Y. "Model for Large Strain Consolidation Under Constant Rate of Strain". International Journal for Numerical and Analytical Methods in Geomechanics, 37(11), pp. 1574-1590. 2013. https:// doi.org/10.1002/nag.2100

[23] Fox, P. J., Pu, H., Christian, J. T. "Evaluation of Data Analysis Methods for the CRS Consolidation Test". ASCE Journal of Geotechnical and Geoenvironmental Engineering, 140(6), pp. 04014020- (1-11). 2014. https://doi.org/10.1061/(ASCE)GT.1943-5606.0001103

[24] Das, B. M. "Numerical solution for one-dimensional consolidation". In: Advanced Soil Mechanics, pp. 300-310. Taylor \& Francis, 2008.

[25] Terzaghi, K., Peck, R. B. "Soil Mechanics in Engineering Practice". Wiley, New York. 1967.

[26] U.S. NAVY. "Soil Mechanics, Foundations, and Earth Structu res". NAVFAC Design Manual DM-7, Washington, D.C. 1971.

[27] Lok, T. M. H., Shi, X. "Consolidation and strength Properties of Macau Marine Clay". Faculty of science and technology, university of Macau. 2008. 